\title{
A possible origin for large aspect angle "HAIR" echoes seen by SuperDARN radars in the $E$ region
}

\author{
J. Drexler ${ }^{1,2}$ and J.-P. St.-Maurice ${ }^{1}$ \\ ${ }^{1}$ Institute of Space and Atmospheric Studies, The University of Saskatchewan, Saskatoon, Saskatchewan, Canada \\ ${ }^{2}$ now at: Department of Earth and Atmospheric Sciences, Cornell University, Ithaca, New York, USA
}

Received: 10 September 2004 - Revised: 18 January 2005 - Accepted: 21 January 2005 - Published: 30 March 2005

\begin{abstract}
Milan et al. (2004) have recently reported on close-range $\mathrm{E}$ region decameter size echoes that seem to be relatively weak, have apparently unusually large aspect angles, and possess Doppler shifts that are slow and are clearly consistent with the ion drift of the medium as opposed to, say, its electron drift or its ion-acoustic speed. We argue that these irregularities are the result of a nonlinear wave conversion process triggered by the nonlocal evolution of decameter Farley-Buneman waves. According to this picture, structures which have weak spontaneous growth rates and are initially field-aligned undergo an evolution of their aspect angle that results in a jump in the aspect angle at some point in time and space. When this takes place, a rapid mode conversion must follow, which takes energy away from a standard twostream signature and converts it either to a strongly damped ion-acoustic mode or to a purely decaying mode, depending on altitude.
\end{abstract}

Keywords. Ionosphere (auroral ionosphere; ionospheric irregularities; plasma waves and instabilities)

\section{Introduction}

Milan et al. (2004) have used the SuperDARN chain of HF radars (e.g. Greenwald et al., 1985) to study high-latitude $\mathrm{E}$ region echoes. They found an unusual class of closerange $\mathrm{E}$ region echoes from an apparent "high-aspect angle irregularity region" (HAIR) that are relatively weak, have unusually high aspect angles, and possess Doppler shifts that are slow and are clearly consistent with the ion drift of the medium as opposed to, say, its electron drift or its ionacoustic speed.

Milan et al. (2004) used an often-quoted approximation of the root to the fluid dispersion relation in order to analyze the results. We show here, however, that this formula is not valid at the large aspect angles considered by the authors.

Correspondence to: J. Drexler

(josef@geology.cornell.edu)
While the resulting frequency appears to be valid, applying the usual root of the fluid dispersion relation at large aspect angles yields strong decay rates which are at odds with the small growth rate approximation that was used to derive it. However, when a dispersion relation valid at large aspect angles is analyzed, it yields the solutions which Milan et al. (2004) expected, namely, modes that move at the ion drift speed in the majority of cases. Nevertheless, this still leaves one important question open, namely: one still has to figure out how the HAIR modes have acquired their energy, since they are purely decaying modes in the first place.

We propose here that the modes are fed by a nonlinear mode-coupling mechanism triggered by the nonlocal evolution of slowly growing primary waves. We show that even initially field-aligned structures evolve to non-zero aspect angles, but that while the mode does not develop large aspect angles as such, it will have a jump in the aspect angle. Even when the aspect angle itself remains relatively small (less than two degrees), this jump has properties that are entirely equivalent to a large aspect angle case. In the nonlinear context, our explanation for the HAIR echoes is similar to the one that was advanced for the "type II" modes uncovered years ago in the equatorial E region (Sudan et al., 1973), in that the modes are linearly stable but are nevertheless excited to detectable levels by nonlinear processes.

\section{The roots of the dispersion relation}

\subsection{Nonlocal generalization of the dispersion equation}

Drexler et al. (2002) have presented a nonlocal description of Farley-Buneman waves based on a standard linearization procedure which can be found in many papers (e.g. Sudan, 1983). The nonlocal effects came from having a variation along the magnetic field of the various parameters that drive the roots of the dispersion relation. Thus, in lieu of a parallel wavevector (or aspect angle) description along the magnetic field (or $z$ ) direction, Drexler et al. (2002) used the 
(a) Frequency

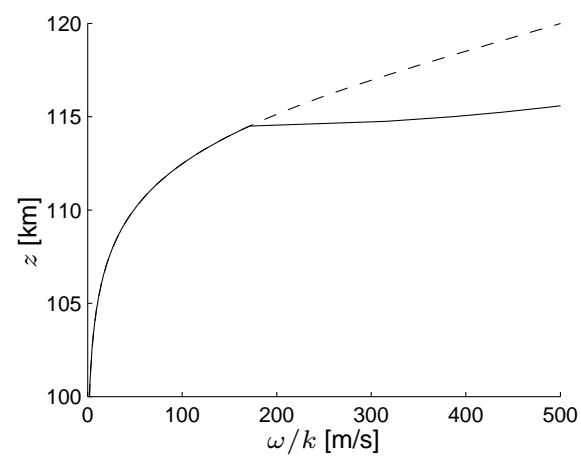

(b) Decay rate

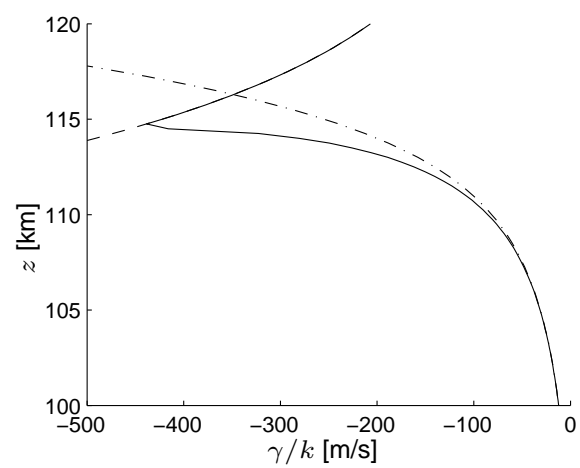

Fig. 1. Panel (a): Profile of the high-aspect angle phase speed $\omega / k$ seen by a ground-based observer (solid) and of the ion drift (dashed). Panel (b): Corresponding plots of the normalized decay rate $\gamma / k$ (solid), as well as of $-v_{i} / 2$ (dashed) and $-k_{\perp}^{2} c_{S}^{2} / v_{i}$ (dash-dotted).

original spatial derivatives for the $z$ direction, while still Fourier analyzing the waves in the plane perpendicular to the magnetic field. From this, they found the following linearized partial differential equation in terms of the perturbed density $\delta n / n_{0}$

$$
\begin{aligned}
& \frac{\partial^{2}}{\partial z^{2}}\left(\frac{\partial^{2}}{\partial t^{\prime 2}}+v_{i} \frac{\partial}{\partial t^{\prime}}+k_{\perp}^{2} c_{s}^{2}\right) \frac{\delta n}{n_{0}} \\
& -\frac{k_{\perp}^{2} v_{e}^{2}}{\Omega_{e}^{2}}\left[\frac{\partial^{2}}{\partial t^{\prime 2}}+v_{i}\left(1+\frac{1}{\Psi_{0}}\right) \frac{\partial}{\partial t^{\prime}}+k_{\perp}^{2} c_{s}^{2}+i \frac{v_{i}}{\Psi_{0}} \boldsymbol{k}_{\perp} \cdot \boldsymbol{v}_{d}\right] \frac{\delta n}{n_{0}}=0(1)
\end{aligned}
$$

where $\partial / \partial t^{\prime}=\partial / \partial t+i \boldsymbol{k}_{\perp} \cdot \boldsymbol{v}_{i}$ represents the time derivative in the frame of reference moving with the ions, at velocity $\boldsymbol{v}_{i}$. The subscripts $e$ and $i$ refer to the electron and ion populations, respectively. The symbols $v_{j}$ and $\Omega_{j}$ are the collision and cyclotron frequencies of species $j, \boldsymbol{v}_{d}=\boldsymbol{v}_{e}-\boldsymbol{v}_{i}$ is the relative electron-ion drift, $c_{s}$ is the ion-acoustic speed, and $\Psi_{0}=\left(v_{e} v_{i}\right) /\left(\Omega_{e} \Omega_{i}\right)$.

This nonlocal description of $\mathrm{E}$ region irregularities allowed Drexler et al. (2002) to describe how coupling with the neutral background forces the instability to evolve in the parallel direction. This coupling is associated with a corresponding parallel group velocity that means waves can saturate by moving through the unstable region until the growth rate decreases to zero. This motion is similar to mechanisms proposed originally by Kaw (1972) and Lee and Kennel (1973), in a different context for the equatorial electrojet. In the present paper, we show that it is not only saturation from a zero-growth condition that produces ion-acoustic modes, but that shocks stemming from a nonlinear evolution of the aspect angle can excite ion-acoustic modes as well.

\subsection{The standard (local) dispersion relation and its roots}

In the event that the collision frequency and/or the background temperature gradients can be neglected, we can use a plane wave decomposition of $\delta n / n_{0}$ along the $z$-direction in order to solve Eq. 1. In that case, we immediately recover the traditional Farley-Buneman dispersion relation for the complex frequency $\omega_{c}^{\prime}$, namely,

$\omega_{c}^{\prime}\left(\omega_{c}^{\prime}+i v_{i}\right)+i \frac{\nu_{i}}{\Psi}\left(\omega_{c}^{\prime}-\boldsymbol{k}_{\perp} \cdot \boldsymbol{v}_{d}\right)-k_{\perp}^{2} c_{s}^{2}=0$

where

$\Psi=\Psi_{0}\left(1+\frac{\Omega_{e}^{2} k_{\|}^{2}}{v_{e}^{2} k^{2}}\right)$

\subsection{The small $\Psi$ limit}

As far as an analysis of the roots of this dispersion relation is concerned, it will be important to note below that a proper perturbation scheme in terms of an expansion based on $\Psi \ll 1$ is the better way to recover the well-known so-called "small growth rate approximation", because the growth rate will not in fact turn out to be small compared to the frequency for the modes we are interested in. To this goal, we just rewrite the previous equation as

$\Psi\left(\omega_{c}^{\prime 2}-k_{\perp}^{2} c_{s}^{2}\right)+i v_{i}\left(\omega_{c}^{\prime}+\Psi \omega_{c}^{\prime}-\boldsymbol{k}_{\perp} \cdot \boldsymbol{v}_{d}\right)=0$

If we now expand in terms of the small parameter $\Psi$ we then assume

$\omega_{c}^{\prime}=\omega_{0}+\Psi \omega_{1}$

From this, we require the following leading order solutions

$\omega_{0}=\boldsymbol{k}_{\perp} \cdot \boldsymbol{v}_{d}$

and

$\Psi \omega_{1}=\frac{\Psi}{i v_{i}}\left(k_{\perp}^{2} c_{s}^{2}-\omega_{0}^{2}-i v_{i} \omega_{0}\right)$

Combining these two solution we then obtain

$\omega_{c}^{\prime}=\boldsymbol{k}_{\perp} \cdot \boldsymbol{v}_{d}(1-\Psi)-i \frac{\Psi}{v_{i}}\left(k_{\perp}^{2} c_{s}^{2}-\omega_{0}^{2}\right)$

For $\Psi \ll 1$, this is essentially the solution that has been repeatedly used in the past (see e.g. Fejer et al., 1984), including by 
Milan et al. (2004). One important point about the derivation, however, is that we do not have to assume that the growth rate, that is, the imaginary part of $\omega_{c}^{\prime}$, is "small" (which is normally implicitly assumed to mean "by comparison to the real part of the frequency"). Instead, the smallness of $\Psi$ itself directs the solution to having a small growth rate in the event that $\boldsymbol{k}_{\perp} \cdot \boldsymbol{v}_{d}$ is large. However, the solution is valid even when $\boldsymbol{k}_{\perp} \cdot \boldsymbol{v}_{d}$ is small. It is important to consider this possibility in modern studies. For instance, in so-called "type II" modes, the wave vector and relative drift tend to be at right angles to one another. In principle, in such cases, a solution based on the "small growth rate approximation" should not be used. However, this inconsistency is removed very easily by using the small $\Psi$ approximation instead of the less general small growth rate approximation.

\subsection{The large $\Psi$ limit}

For the work at hand it is also very important to look at the approximate solutions in terms of large magnitudes in $\Psi$ when considering the large aspect angle limit, as Milan et al. (2004) were attempting to do. According to Eq. 3 the value of $\Psi$ rapidly becomes greater than 1 as the aspect angle increases above $0.5^{\circ}$, for the ionospheric situations of interest. As a result, we find that, to leading order, at large aspect angles, the $1 / \Psi$ term disappears from Eq. (2) so that the dispersion relation now becomes

$\omega_{c}^{\prime}\left(\omega_{c}^{\prime}+i v_{i}\right)-k_{\perp}^{2} c_{s}^{2} \approx 0$

We find the solution to be that of a damped harmonic oscillator, namely,

$\omega_{c}^{\prime}= \pm \sqrt{k_{\perp}^{2} c_{s}^{2}-v_{i}^{2} / 4}-i v_{i} / 2$

When $v_{i} / 2$ is small by comparison to $k_{\perp} c_{s}$ (typically, above $115 \mathrm{~km}$ for decameter structures) these modes have a damped ion-acoustic signature and might be identified by experimentalists as being so-called "type I" modes in spite of their large aspect angles.

For $v_{i} / 2>k_{\perp} c_{s}$ on the other hand (below approximately $115 \mathrm{~km}$ ), the large-aspect angle dispersion relation has, instead, a purely damped, non-oscillating character, namely,

$\operatorname{Re}\left(\omega_{c}^{\prime}\right)=0 \quad \operatorname{Im}\left(\omega_{c}^{\prime}\right)=-v_{i} / 2 \pm \sqrt{v_{i}^{2} / 4-k_{\perp}^{2} c_{s}^{2}}$

In the limit of very large collision frequencies this yields the heavily damped solution $\gamma=\operatorname{Im}\left(\omega_{c}^{\prime}\right)=-v_{i}$ as well as the more weakly-damped root given by

$\gamma \approx-\frac{k_{\perp}^{2} c_{s}^{2}}{v_{i}}$

The bottom line is that if, well below $115 \mathrm{~km}$ (say $105 \mathrm{~km}$ ), a large aspect angle decameter mode is excited (by whatever mechanism) it will have one root that is relatively weakly damped. A ground-based observer will see a weak Doppler shift associated with that mode, since it will be created in the neutral frame of reference. In other words, we will observe a frequency given by

$\omega=\operatorname{Re}\left(\omega_{c}^{\prime}\right)+\boldsymbol{k}_{\perp} \cdot \boldsymbol{v}_{i}=\boldsymbol{k}_{\perp} \cdot \boldsymbol{v}_{i}$

We claim that these are the HAIR modes that were observed by Milan et al. (2004) since these authors were adamant about the fact that they had observed larger aspect angle modes with a phase velocity that matched the ion drift along the line of sight, just as indicated by Eq. (13).

Profiles of $\omega$ and $\gamma$ (not using the Eq.(12) approximation) are shown in Fig. 1. As expected, below approximately $115 \mathrm{~km}$ (where $v_{i} / 2=k_{\perp} c_{s}$ ), the frequency follows the ion drift, and has an additional component from Eq. (10) above this altitude that rapidly goes to $k_{\perp} c_{s}$. Similarly, the growth rate follows the small magnitudes from Eq. (12) below $110 \mathrm{~km}$, but switches to $v_{i} / 2$ above $115 \mathrm{~km}$.

\subsection{How nonlocal effects generate large aspect angle solu- tions}

Thus far we have shown that, in the context of the standard local dispersion relation, if large enough aspect angles are generated in the $100 \mathrm{~km}$ to $110 \mathrm{~km}$ region they will match the observed properties of the HAIR echoes. It is not clear in that context, however, how modes could be generated with these large aspect angles, since they are strongly damped and cannot be generated directly.

The answer to the mystery seems to be rooted in the nonlocal evolution of decameter structures. We will show in the next section that one consequence of the nonlocal evolution undergone by slowly growing structures is that they evolve a large derivative in their parallel wave vector (or aspect angle). If we then return to our first equation and explore what happens under conditions for which the second $z$-derivative is trying to become very large (equivalent to a study of large aspect angle solutions in standard local theory, but not for the same physics) we find that the first term in Eq. (1) has to dominate the second term. However, since the two terms must add up to zero, this means that the first term has to go to zero by itself. Not only that, but it is the term whose second $z$-derivative is taken that has to go to zero. When that happens, however, the equation being solved becomes identical to Eq. (9).

We conclude from this that the HAIR echoes are generated because decameter structures evolve discontinuities in their aspect angles, which then feed purely decaying modes below $110 \mathrm{~km}$ altitude. This means that the Doppler shift of the modes will match the line of sight velocity of the ions. Furthermore, in the likely event that energy is fed to the modes at a rate faster than their natural decay rate (which seems likely in mode-coupling situations such as this), the spectral width should be comparable to the magnitude of the growth rate, given by Eq. (12). 
(a) Aspect angle

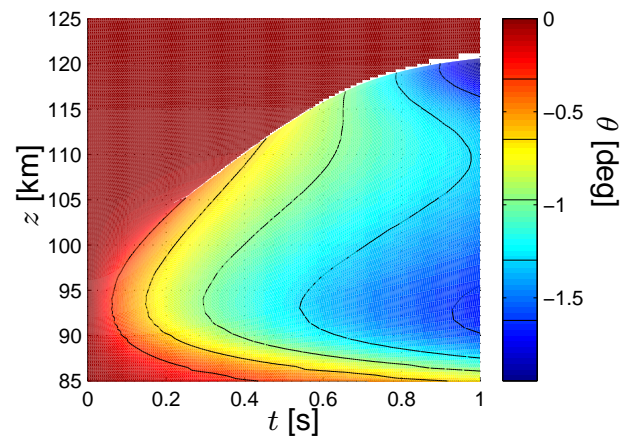

(b) Parallel group velocity

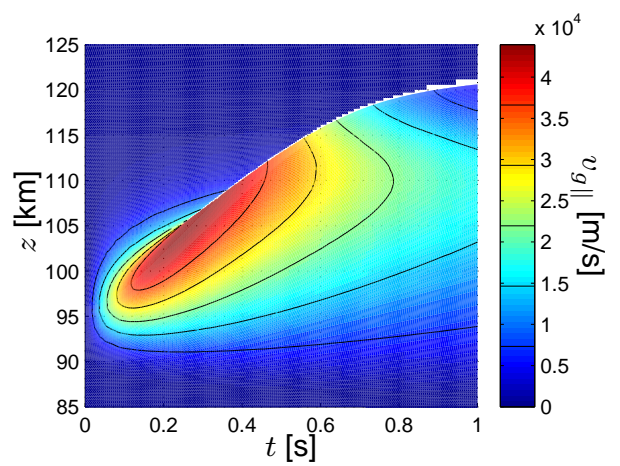

Fig. 2. Typical aspect angle and group velocity evolution from initial condition of $k_{\|}=0$. Panel (a): Aspect angle in degrees shown as function of altitude and time. Color indicates the value of the aspect angle, with black contours corresponding to the horizontal lines in the color bar to the right. Panel (b): Corresponding group velocity for $E / B=1000 \mathrm{~m} / \mathrm{s}$, in the same format. Positive values represent motion to higher altitudes.

\section{Nonlocal theory}

Drexler et al. (2002) have solved the nonlocal FarleyBuneman generalization provided by Eq. (1) using a WKBlike description of the density perturbation, namely, a perturbation of the form

$$
\frac{\delta n}{n_{0}}(z, t)=A(z, t) \exp [i S(z, t)]
$$

where $A$ and $S$ were considered real. Drexler et al. (2002) used a multi-timing multi-scaling expansion of Eq. (1) to describe the evolution of the system. From the fastest time and spacial scales they uncovered that the following expression for the generalized wave frequency, $\partial S / \partial t \equiv \omega$, had to replace the root of the ordinary (local) dispersion relation

$\omega(z, t)=\frac{\boldsymbol{k}_{\perp} \cdot \boldsymbol{v}_{d}(z)}{1+\Psi(z, t)}+\boldsymbol{k}_{\perp} \cdot \boldsymbol{v}_{i}(z)$

The similarity with the local frequency expression (see e.g. Fejer et al., 1984) is obvious, but notice that unlike the eigenvalue problem, the generalized frequency is now allowed to be a function of time and space.

From the next order equation in time and space Drexler et al. (2002) were able to obtain an equation to describe the change in the amplitude of the structure. They also showed that this second equation could be obtained by invoking conservation of wave-action. Essentially, wave action is defined as wave energy $U$ divided by the so-called "intrinsic" frequency $\omega^{\prime}$. Once local growth or decay are taken into account, wave action should be conserved for motion through a slowly changing background (e.g. Bretherton and Garret, 1969; Andrews and McIntyre, 1978). At E region altitudes, wave energy is essentially given by the perturbed kinetic energy of the ions, i.e.,

$U=\frac{1}{2} n_{0} m_{i} \frac{\omega^{\prime 2}}{k^{2}}|A|^{2}$

Since energy is carried by the group velocity, conservation of wave action (or, more precisely, balance of wave action in the presence of a local growth term $\gamma$ ) then leads to an equation for the wave amplitude $A(z, t)$,

$$
\begin{aligned}
& \frac{\partial A}{\partial t}+v_{g \|} \frac{\partial A}{\partial z}= \\
& \quad \gamma A-\frac{A}{2}\left[\frac{v_{g \|}}{k_{\|}} \frac{\partial k_{\|}}{\partial z}-\frac{2 v_{g \|}}{1+\Psi} \frac{\partial \Psi}{\partial z}+\frac{2 v_{g \|}}{\boldsymbol{k}_{\perp} \cdot \boldsymbol{v}_{d}} \frac{\partial \boldsymbol{k}_{\perp} \cdot \boldsymbol{v}_{d}}{\partial z}\right]
\end{aligned}
$$

where $\gamma$ is the usual local growth rate for the instability. Notice once again that we are now using a generalization of the parallel wave vector, which is defined by $k_{\|}=-\partial S / \partial z$ and that, as a result, $k_{\|}$can also be a function of time and space, unlike what is found in a plane wave decomposition.

Equation (17) clearly shows how local and nonlocal processes compete. For instance, when local processes dominate only the first terms on each side of the equation are involved and we have standard linear instability theory. The converse is that nonlocal effects become important when the growth rate is unable to compete with terms that involve the parallel group velocity $\boldsymbol{v}_{g \|}$. The latter is given by the usual definition as

$v_{g \|}=\hat{\boldsymbol{B}} \cdot \boldsymbol{v}_{g}=\hat{\boldsymbol{B}} \cdot \nabla_{k} \omega=\frac{\partial \omega}{\partial k_{\|}}$

where $\hat{\boldsymbol{B}}=\boldsymbol{B} / \boldsymbol{B}$. Together with Eqs. (3) and (15), we then find that

$v_{g \|}=-2 \frac{\omega^{\prime}}{k} \frac{\Psi_{0}}{(1+\Psi)} \frac{k_{\|}}{k} \frac{\Omega_{e}^{2}}{v_{e}^{2}}$

can easily be of the order of 10 to $20 \mathrm{~km} / \mathrm{s}$ at small but nonzero aspect angles.

A central point in the description of the evolution of the structures is that the aspect angle must evolve constantly in time and space. This evolution has to simply be described by the so-called Whitham relation, namely,

$\frac{\partial k_{\|}}{\partial t}=-\frac{\partial \omega}{\partial z}$ 
Above $105 \mathrm{~km}, \partial \Psi / \partial z$ is small, and therefore so is $\partial \omega / \partial z$ (see Eq. 15). As a result, there is relatively little evolution in $k_{\|}$above $105 \mathrm{~km}$. At lower altitudes however, $\Psi$ is no longer negligible with the result that $\omega$ has a large gradient in the $z$ direction (with $\omega$ itself being larger at higher altitudes). This means that $k_{\|}$has to become increasingly negative as time goes on, as can readily be seen from Eq. (20).

One consequence of the evolution of $k_{\|}$is the corresponding evolution of the parallel (nearly vertical) group velocity $v_{g \|}$. Since $k_{\|}$stays small above $105 \mathrm{~km}$, so does the group velocity there, and therefore the upper parts of the wave have practically no vertical motion. At lower altitudes however, $k_{\|}$decreases monotonically, and the group velocity grows larger. This means that the lower parts of the wave will move upwards, eventually colliding with its upper parts. As this happens, the solution ultimately develops a shock, as the aspect angle and associated group velocity evolve an increasingly strong gradient, and, in the end, a discontinuity.

The evolutions of the aspect angle and group velocity are illustrated in Fig. 2 for ionospheric parameters of interest. In this particular example, because $\omega$ is a strong function of $z$ below $115 \mathrm{~km}$, the aspect angle $k_{\|} / k$ reaches more than $1^{\circ}$ in $1 \mathrm{~s}$. The corresponding group velocity reaches more than 40 times $E / B$. However, most important for the problem at hand is the fact that after approximately $0.3 \mathrm{~s}$, a discontinuity forms in the aspect angle. It can be identified as the sharp transition from almost-zero aspect angles and group velocities above to larger values below.

While the production of a large jump in the derivative of the aspect angle guarantees that the structure will be feeding damped ion-acoustic modes above $115 \mathrm{~km}$, and purely damped modes below that height, we still need to know if the new modes are fed enough energy for the process to matter. To this goal we have to look at the amplitude reached by the structures just prior to the shock, using the solution to Eq. (17). Such a solution is shown in Fig. 3, calculated for the conditions associated with the aspect angle and parallel group velocity plots presented in the previous figures. We can clearly see from this calculation (and many more cases studied by Drexler et al., 2002) that the structures can indeed gain a substantial amplitude before feeding their energy to the large aspect angle modes. In the example presented here, the largest amplitudes being fed to the shocked modes are seen around $0.5 \mathrm{~s}$ after the initial perfectly field-aligned perturbation. At that point we should note that the aspect angle is larger than at earlier times, but it is not really out of the ordinary. However, this does not stop the derivative of the aspect angle (second derivative of the phase with respect to $z$ ) from taking a large value. This point is illustrated more precisely with Fig. 4, where the aspect angle evolution is shown as a function of altitude for different times. As a word of caution, we note, however, that whether or not the same aspect angle should be found in the damped modes that receive the energy is another question that cannot be addressed with the limited tools that we have used here.

We can summarize the physical principles at work in the nonlocal evolution as follows: 1) the inhomogeneity in the

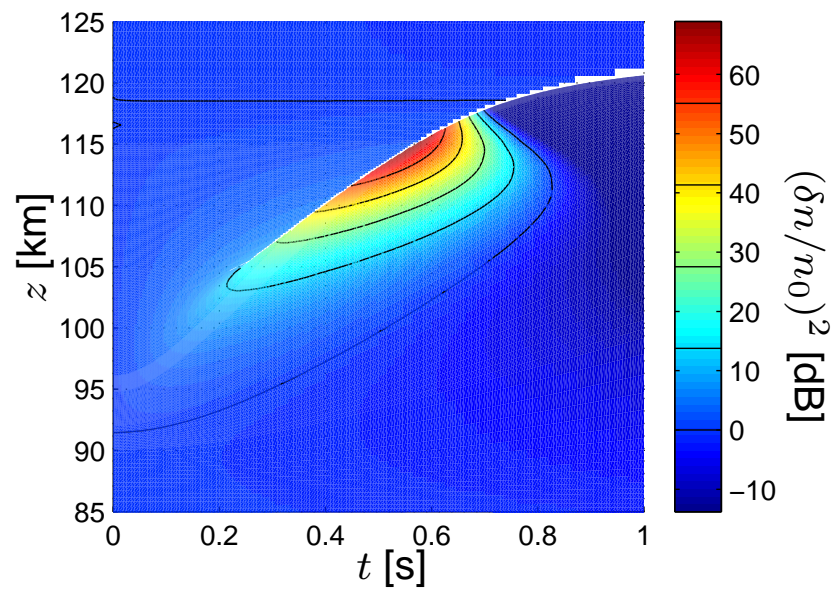

Fig. 3. Wave amplitude corresponding to the parameters of Fig. 2. The color scale represents the amount of growth in $\mathrm{dB}$ relative to the initial value at $t=0 \mathrm{~s}$.

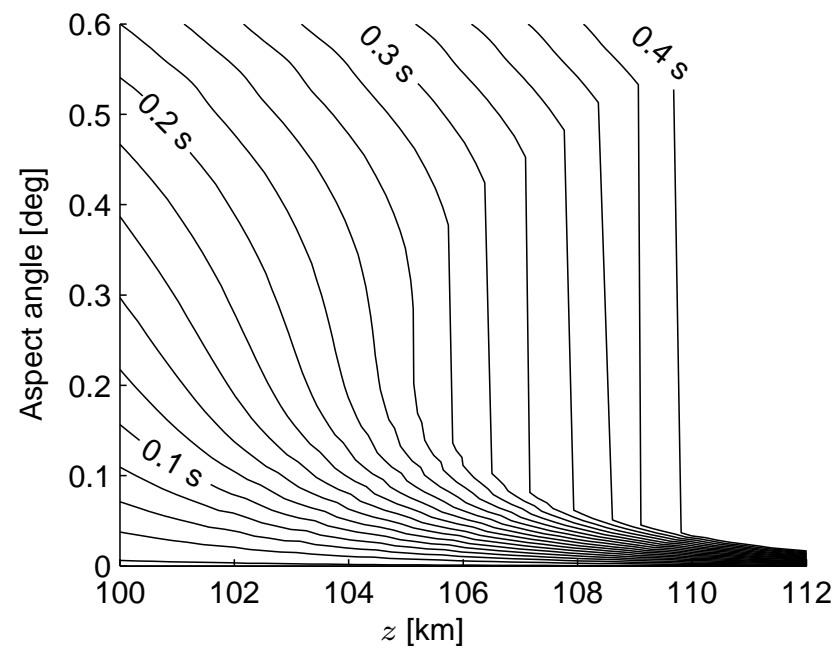

Fig. 4. Aspect angle profiles from $t=0 \mathrm{~s}$ (left-most curve) to $t=0.4 \mathrm{~s}$ (right-most curve) at $0.02 \mathrm{~s}$ intervals. After $\sim 0.3 \mathrm{~s}$ a strong gradient and later a jump in the aspect angle develops.

neutral atmosphere causes an evolution of the aspect angle of a structure (through $\partial \omega / \partial z$ ); 2) this in turn causes the lower altitude parts of the structure to move upwards and collide with the largely stationary upper parts; 3) this collision leads to a shock, i.e., a discontinuity, which affects the aspect angle, the group velocity, the amplitude and all other derived quantities; 4) as far as where the energy goes once the shock is triggered, all we can state at this point is that it has to be fed to the damped modes that we have identified in the previous section but the question of what aspect angle these modes should have cannot be addressed with the tools that we have used here. 


\section{Conclusions}

Starting from the work of Drexler et al. (2002) we have demonstrated how the inhomogeneity in the neutral atmosphere forces slowly growing E region decameter instabilities to undergo an evolution in their aspect angle so that they develop an aspect angle discontinuity or shock. When this happens, the wave must switch to a different mode with zero frequency (or slightly larger above $115 \mathrm{~km}$ ) in the ion frame of reference. A ground-based observer would therefore see radar echoes from below $115 \mathrm{~km}$ with a Doppler shift near the line-of-sight component of the ion drift, similar to what would be seen when observing large aspect angle waves.

While we have been able to identify the Doppler velocity and damping rate of the new modes triggered by the evolution of a discontinuity in aspect angle, we are unable at this point to predict what the aspect angle of the secondary echoes should be. This is because with the tools that we have been able to use, these echoes may appear at any aspect angle, their aspect angle being in fact undefined. All we can tell is that before the mode conversion, the aspect angle is within the usual range expected for the nearly-field-aligned irregularities that have been observed over the years. Also notice that the perpendicular component of the wave vector, $\boldsymbol{k}_{\perp}$, will remain in the direction of the original wave.

Since their origin is an unstable Farley-Buneman wave, it is to be expected that the new crashed modes will start out with fairly large amplitudes. Having a moderately large negative growth rate, these modes are then damped fairly quickly. It is possible in fact that the decay rate given by Eq. (12) could be comparable to the spectral width of the observed modes, if the energy happens to be fed quickly, as we might expect from mode-coupling episodes.

We conclude that the Drexler et al. (2002) nonlocal model analysis can be used to provide an explanation for the echoes observed by Milan et al. (2004). The model can be used to explain the Doppler characteristics as well as to obtain a mechanism to provide energy to the damped modes.
Acknowledgements. This work has been supported by a Canadian National Science and Research Engineering Council grant.

Topical Editor M. Lester thanks J. Sahr and another referee for their help in evaluating this paper.

\section{References}

Andrews, D. G. and McIntyre, M. E.: On wave-action and its relatives, J. Fluid Mech., 89, 647-664, 1978.

Bretherton, F. P. and Garret, C. J. R.: Wave trains in inhomogeneous moving media, Proc. Roy. Soc. A, 302, 529-554, 1969.

Drexler, J., St.-Maurice, J.-P., Chen, D., and Moorcroft, D. R.: New insights from a nonlocal generalization of the Farley-Buneman instability problem at high latitudes, Ann. Geophys., 20, 2003 2025, 2002,

SRef-ID: 1432-0576/ag/2002-20-2003.

Fejer, B. G., Providakes, J. F., and Farley, D. T.: Theory of plasma waves in the auroral E region, J. Geophys. Res., 89, 7487-7494, 1984.

Greenwald, R. A., Baker, K. B., Hutchins, R. A., and Hanuise, C.: An HF phased-array radar for studying small-scale structure in the high-latitude ionosphere, Radio Sci., 20, 63-79, 1985.

Kaw, P. K.: Wave Propagation Effects on Observation of Irregularities in the Equatorial Electrojet, J. Geophys. Res., 77, 13231326, 1972.

Lee, K. and Kennel, C. F.: Effects of propagation parallel to the magnetic field on the type I electrojet irregularity instability, Planet. Space Sci., 21, 1339-1343, 1973.

Milan, S. E., Lester, M., Yeoman, T. K., Robinson, T. R., Uspensky, M. V., and Villain, J.-P.: HF radar observations of high-aspect angle backscatter from the E-region, Ann. Geophys., 22, 829847, 2004,

SRef-ID: 1432-0576/ag/2004-22-829.

Sudan, R. N.: Unified Theory of Type I and Type II Irregularities in the Equatorial Electrojet, J. Geophys. Res., 88, 4853-4860, 1983.

Sudan, R. N., Akinrimisi, J., and Farley, D. T.: Generation of smallscale irregularities in the equatorial electrojet, J. Geophys. Res., 78, 240-248, 1973. 